\title{
Le partage des responsabilités entre les acteurs de la politique de l'emploi. Vers une configuration multipolaire (1945-1973)
}

The share responsabilities betwen actors of employment policies : the creation of a multi-polar "configuration of actors" (1945-1973)

\section{Jacques Freyssinet}

\section{OpenEdition}

Journals

Édition électronique

URL : http://journals.openedition.org/travailemploi/4574

DOI : 10.4000/travailemploi.4574

ISSN : $1775-416 \mathrm{X}$

Éditeur

DARES - Ministère du Travail

Édition imprimée

Date de publication : 30 mars 2007

Pagination : 37-46

ISSN : 0224-4365

Référence électronique

Jacques Freyssinet, "Le partage des responsabilités entre les acteurs de la politique de l'emploi. Vers une configuration multipolaire (1945-1973)», Travail et Emploi [En ligne], 110 | Avril-Juin 2007, mis en ligne le 15 juin 2009, consulté le 01 mai 2019. URL : http://journals.openedition.org/ travailemploi/4574; DOI : 10.4000/travailemploi.4574 


\title{
Le partage des responsabilités entre les acteurs de la politique de l'emploi Vers une configuration multipolaire (1945-1973)
}

\author{
Jacques Freyssinet $(*)$
}

\begin{abstract}
Au lendemain de la Seconde Guerre mondiale, la logique de l'économie dirigée fait de la politique de la main-d'œuvre la responsabilité de l'État. Mais une évolution profonde du mode de gouvernance va se développer progressivement, caractérisée par le retour des acteurs économiques et sociaux. L'article montre comment la complexification des acteurs était en germe dès les années 1945 à 1973 et contribue ainsi à éclairer les enjeux actuels. À partir des travaux du Commissariat général du Plan notamment, il montre quelles ont été les inflexions de cette politique, trop longtemps présentée comme un développement linéaire: l'évolution n'engendrera pas un partage clair des compétences, mais un empilement d'institutions et de dispositifs hétérogènes qui ne réussira à fonctionner que tant que le modèle de croissance qui avait servi de base à son édification se maintiendra.
\end{abstract}

La période qui va de 1945 à 1973 est souvent présentée comme celle de l'«invention» de la politique de l'emploi. Cette politique a fait l'objet de nombreuses analyses qui portaient sur son contenu (1); en revanche, peu de travaux s'intéressent aux responsabilités respectives exercées par ses différents acteurs.

Bernard Lacroix et Vincent Merle ont insisté sur la nécessité d'adopter une approche archéologique de la genèse des institutions sociales (MerLe, 1987; Merle, Lacroix, 1988). Cette approche s'oppose, d'une part, à une vision naturaliste qui prétend décrire une progression cumulative et linéaire des droits reconnus ou des instruments mobilisés, d'autre part, à une vision instrumentaliste, qui ne voit dans les modes successifs d'intervention de l'État que les figures variées d'une même fonction régulatrice. La politique de l'emploi fournit un bon exemple de l'intérêt d'une telle approche généalogique. Elle n'est pas le lieu d'une progression cumulative et linéaire,

(*) Professeur émérite à l'université Paris I, président du Conseil scientifique du Centre d'études de l'emploi. Adresse e-mail: jacques.freyssinet@mail.enpc.fr

(1) Nous avons étudié le contenu des politiques de la maind'œuvre et de l'emploi dans un autre texte, L'émergence des politiques de l'emploi (1945-1973) (FreYsSINET, 2006). Un document de travail sous le même titre, accessible sur le site du Centre d'études de l'emploi, contient des analyses plus détaillées et une bibliographie plus complète. Une première version a bénéficié d'observations critiques et de compléments d'information de la part de Dominique Balmary, Jacques Delors, Robert Fraisse, Jean-Marcel Jeanneney, Marie-Thérèse Join-Lambert, et Gabriel Mignot. Ils en sont vivement remerciés, mais, comme il est de règle, ne sont en rien engagés par le contenu de ce texte. mais marquée par des ruptures dans le choix des objectifs et des outils. Elle n'est pas la manifestation d'une fonction régulatrice inchangée de l'État, mais de mutations successives dans la conception des responsabilités de l'État et du partage des rôles avec d'autres acteurs. En tant qu'élément constitutif du processus de mobilisation et d'allocation des forces de travail, la politique de l'emploi reflète et tente d'infléchir, dans des rapports de forces évolutifs, les modes de régulation des relations collectives de travail. Elle est le produit complexe de l'inertie des trajectoires et de ruptures associées à des phases de crise. Elle ne peut donc être comprise que dans le cadre d'une dynamique historique qui permette de saisir les interactions entre les stratégies des différents acteurs concernés.

Avant d'examiner les différents types de rapports qui s'établissent au cours de la période 1945-1973 entre ces acteurs (concertation, contractualisation, délégation), nous rappellerons les étapes de création d'une politique de la main-d'œuvre, qui ont déterminé l'«héritage» reçu au terme de la Seconde Guerre mondiale, puis nous tenterons, en conclusion, de dégager les éléments de continuité et de rupture qui s'observent dans les évolutions observées à partir de la seconde moitié de la décennie 1970.

\section{La genèse chaotique d'une configuration d'acteurs}

De manière schématique, il est possible d'opposer, dans un premier temps, un lent mouvement 


\section{Encadré 1}

\section{Nature des sources institutionnelles mobilisées}

a) Pour l'analyse des positions des différents acteurs, nous utilisons de manière privilégiée les rapports du Commissariat général du plan (CGP) (1) et, en particulier, les travaux de préparation des plans successifs (CGP, 1947, 1954, 1958, 1961, 1966 et 1971; cf. aussi: DeCOUfLÉ, 1990; JACHIET, 1981 et sur certains aspects du fonctionnement des commissions du Plan: DASSETTO, 1972; HaYWARD, 1967; MASSÉ, 1964). Les commissions du Plan sont un lieu de confrontation entre les différents acteurs publics concernés, avec une participation souvent active des acteurs sociaux.

b) Les positions adoptées par les acteurs économiques et sociaux sont régulièrement rassemblées dans les rapports et avis que le Conseil économique et social consacre aux plans successifs, ainsi qu'à différents aspects de la politique de l'emploi. L'expression de chacun des groupes composant le Conseil sur le contenu des avis offre une source régulière pour suivre l'évolution de leurs analyses et de leurs priorités.

c) En revanche, on ne dispose, pour cette période, que d'une information publique réduite sur les débats d'orientation qui ont pu se dérouler au sein du ministère du Travail. La Revue française du travail publie surtout des textes à caractère informatif (mis à part les rapports des commissions de la main-d'œuvre du Plan). Les articles non descriptifs sur la politique de l'emploi sont très peu fréquents et généralement confiés à des chercheurs (Roux, 1949, Dahano, 1971; Gillard, 1974). Les documents de prise de position sur le fond sont rares (à titre d'exception, cf. ChAzeLle, 1964 - non publié). Un travail sur les archives du ministère apporterait peut-être une vision différente.

(1) Le sigle CGP: il désigne l'institution successivement dénommée Commissariat général du plan de modernisation et d'équipement, puis Commissariat général du plan d'équipement et de la productivité, puis Commissariat général du plan.

de renforcement des instruments d'action sur l'emploi avec une montée des responsabilités de l'État, mouvement qui trouve son apogée au lendemain de la Seconde Guerre mondiale, puis, dans un deuxième temps, une diversification des modes de gouvernance qui accompagne la reconnaissance du pluralisme des acteurs de la politique de l'emploi.

\section{L'intervention publique sur l'emploi: émergence des dispositifs et des acteurs}

Dès la fin du XIX ${ }^{\mathrm{e}}$ siècle, avec la création en 1891 du Conseil supérieur du travail, puis de l'Office du travail, un débat est ouvert sur la nature des responsabilités que doit exercer l'État dans le fonction- nement du marché du travail et dans la gestion des déséquilibres qui s'y manifestent. En permanence, la question se pose du partage des compétences avec les agents économiques privés, avec les organisations patronales et syndicales, ainsi qu'avec les collectivités territoriales. Jusqu'à la Seconde Guerre mondiale, le processus d'apprentissage se révèle chaotique. D'une part, il cherche à répondre aux transformations de long terme qui affectent le fonctionnement des économies de marché; d'autre part, il est à deux reprises bouleversé par le choc des guerres mondiales (2).

Le fonctionnement de l'économie de marché est soumis à un ensemble de mouvements cycliques qui vont des fluctuations saisonnières d'activité aux phases de récession économique généralisée, en passant par des crises sectorielles. Sauf la brève exception de 1936, l'État ne se reconnaît pas une responsabilité de régulation économique globale. Les dispositifs s'organisent autour du traitement du chômage. Dès 1905, des subventions sont accordées aux caisses privées (le plus souvent syndicales) d'assurance-chômage, tandis que la loi réglemente l'activité des bureaux de placement. Plus tard, la fonction d'indemnisation est élargie en encourageant l'initiative des communes et départements, avec un financement complémentaire de l'État. Dans les périodes de forte récession, un recours aux chantiers de travaux publics est préconisé, toujours à l'initiative des collectivités territoriales et avec l'aide de l'État. Il s'agit donc essentiellement de gestion du chômage et marginalement d'action sur l'emploi. L'exception vient du programme du Front populaire, dont le premier gouvernement Blum amorce la réalisation. Mêlant des inspirations diverses (premières influences keynésiennes, expériences de Roosevelt, mouvement «planiste»), le programme cherche à lutter contre le chômage en combinant une action de relance macroéconomique (investissements publics d'infrastructure, augmentations négociées des salaires...) et une politique de partage du travail (principalement les « 40 heures»). La tentative est de brève durée, mais annonce bien des débats de la période d'après-guerre.

Les deux guerres mondiales ont été un facteur, au moins aussi important, d'innovation en matière de politique de main-d'œuvre. Elles sont d'abord marquées par de brèves, mais brutales poussées de chômage lors du début des hostilités (désorganisation de la production), puis lors de leur suspension ou cessation (reconversion des productions, afflux des démobilisés ou des prisonniers libérés). Elles engendrent surtout des périodes de pénurie aiguë de main-

(2) Pour une présentation d'ensemble de la période, voir principalement: DESMARETS, 1946 (première et deuxième parties); DANiel, Tuchszirer, 1999 (chapitres I à VII); VIET, 2006. $C f$. aussi Maclouf, 2006; Decouflé, 1982, 1985 et 1988; JoINLAMBERT et alii, 1997 (principalement, chapitres 1, 3 et 7). 
d'œuvre pour répondre soit aux besoins de l'effort de guerre, soit aux exigences de l'occupant. Il en résulte la mise en place, dès la Première Guerre mondiale, d'une véritable planification(3) de la mobilisation et de l'allocation des ressources de main-d'œuvre en fonction d'objectifs nationaux prioritaires. La période de la Seconde Guerre mondiale voit, outre l'éphémère expérience d'un Commissariat à la lutte contre le chômage, la mise en place d'un dispositif intégré de politique de la main-d'œuvre au sein du ministère du Travail: création d'offices régionaux et départementaux du travail (4), fusion des fonctions d'indemnisation et de placement, contrôle rigoureux du recours à la main-d'œuvre coloniale ou étrangère, procédures d'affectation impérative à certaines activités prioritaires, développement de l'apprentissage et des centres de formation professionnelle des adultes, etc.

\section{“Étatisation» de la politique de l'emploi ou retour au pluralisme des acteurs?}

Lorsque Jacques Desmarets (5) publie, au premier trimestre 1946, La politique de la main-d'œuvre en France, il part de l'analyse de ces expériences pour proposer un schéma global de politique de la main-d'œuvre en économie dirigée. Cette politique «a pour objet d'adapter aussi exactement que possible les ressources humaines d'une Nation à ses besoins généraux et plus spécialement à ses besoins économiques» (DeSMARETS, 1946, p. 1). Elle est «un fragment d'une politique économique générale» (op. cit., p. 2) à laquelle elle est subordonnée. Elle devrait relever d'un secrétariat à la main-d'œuvre rattaché au ministère de l'Économie. À partir d'un tableau des besoins et des ressources par métiers et par marchés du travail locaux, celui-ci établirait un plan d'utilisation de la main-d'œuvre. Il regrouperait les fonctions d'orientation et de formation professionnelle des jeunes et des adultes, de placement, d'adaptation du travail aux hommes et d'assistance aux travailleurs sans emploi.

Ainsi, en un demi-siècle, les responsabilités exercées par les différents acteurs se sont-elles profondément transformées. Au départ, l'État ne se reconnaît qu'un modeste droit d'intervention sur le marché du travail au moyen des fonctions de contrôle des intermédiaires privés du placement et d'incitations financières aux organismes d'indemnisation du chômage créés par les syndicats, puis les collectivités territoriales. Au lendemain de la guerre, la logique de l'économie dirigée fait de la politique de la main-d'œuvre une incontestable responsabilité de l'État. Elle a pour fonction de répondre aux objectifs de croissance. Sa mise en œuvre sera

(3) Même si le mot n'est pas employé.

(4) Ils seront rebaptisés «directions» après la guerre.

(5) V. VIET, op. cit. précise que Jacques Desmarets fut, sous Vichy, chargé de mission auprès du commissaire à la lutte contre le chômage, puis directeur de cabinet au commissariat à la main-d'œuvre française en Allemagne. assurée, dans ce cadre, par le ministère du Travail. Les acteurs autres que l'État sont marginalisés.

La période 1945-1973 verra, au contraire, une lente évolution du mode de gouvernance dans le sens d'une restauration d'un pluralisme d'acteurs. Nous en distinguerons trois modalités, non selon un ordre chronologique, mais selon un degré croissant de partage des responsabilités entre l'État et d'autres acteurs économiques et sociaux(6). La modalité la plus modeste correspond à la création d'instances tripartites de consultation ou de concertation. Une deuxième étape est franchie lorsque l'utilisation de dispositifs de la politique de l'emploi est conditionnée à la signature de conventions entre l'État et des acteurs privés. Enfin, l'État semble renoncer à son pouvoir lorsqu'il délègue à la négociation collective la responsabilité de fixer certaines modalités de la politique de l'emploi, voire de créer des institutions paritaires chargées de les mettre en œuvre(7).

\section{Concertation ou consultation?}

Un premier degré de reconnaissance du pluralisme des acteurs résulte de l'introduction d'instances tripartites qui organisent un dialogue au sein des institutions publiques. Il faut distinguer entre les questions qui relèvent de la place de la politique de l'emploi au sein de la politique économique et sociale globale, pour lesquelles le Commissariat général du plan joue un rôle prédominant, et celles qui concernent les responsabilités propres du ministère du Travail, où l'on ne dépasse guère le stade consultatif en dépit de quelques amorces de tripartisme de gestion.

Dans l'immédiat après-guerre, le Plan est un outil de mobilisation pour la reconstruction de l'économie nationale. Il devient ensuite le vecteur de la modernisation; il doit gérer les problèmes de restructuration, puis d'inflation et de compétitivité, enfin, les premières poussées de chômage (JACHIET, 1981). Les travaux des commissions de préparation des plans, par exemple, ceux de la Commission de la main-d'œuvre, devenue à partir du VIe plan la Commission emploi (CGP, 1944, 1954, 1958, 1961, 1966, 1971), constituent un moyen privilégié pour convaincre les acteurs sociaux de la pertinence ou de la nécessité des choix proposés, pour susciter leur adhésion ou évaluer leurs résistances, pour chercher des bases de compromis, explicites ou implicites. Bien des auteurs et bien des acteurs se sont interrogés sur la fonction réelle de telles procédures (8), mais il n'y a là rien qui soit propre au domaine de

(6) Nous ne traiterons pas des collectivités territoriales qui, jusqu'aux lois de décentralisation, ne jouent en ce domaine qu'un rôle marginal.

(7) Sur les sources institutionnelles utilisées, $c f$. l'encadré 1.

(8) On en trouve des illustrations significatives dans Dassetto, 1972 et HaYWARD, 1967. 
l'emploi, même si celui-ci occupe une place importante dans les débats de ces instances. Avec des hauts et des bas selon la conjoncture politique, il n'est pas douteux que le Plan ait été, dans la période, un lieu de discussions libres et approfondies (9).

La consultation est institutionnalisée, au sein du ministère du Travail, par la création, en 1963, du Comité supérieur de l'emploi. Celui-ci, de composition tripartite, a compétence générale sur la politique de l'emploi et doit, de plus, donner des avis sur le choix des critères ou le contenu de certaines conventions de mise en œuvre du Fonds national de l'emploi (FNE) ( $c f$. infra). À l'échelle déconcentrée, apparaissent en 1967 les comités régionaux de la formation professionnelle et de l'emploi, complétés par des comités départementaux. De composition tripartite, ils ont une compétence consultative aussi vaste qu'imprécise. Il ne semble pas qu'existent des études portant sur le fonctionnement de ces organismes. De l'avis général des acteurs sociaux qui y ont participé, les comités constituaient pour eux un lieu utile de recueil d'informations, parfois une tribune pour exposer leurs inquiétudes ou leurs revendications, mais ils sont sceptiques quant à l'influence exercée par la consultation sur les pouvoirs publics (10). Tout au plus, ceux-ci s'assuraient-ils que certaines de leurs décisions ne soulèveraient pas d'opposition trop générale ou trop violente.

Une situation juridiquement différente est créée lorsque les acteurs sociaux sont présents, aux côtés des représentants de l'État, dans les instances qui ont la responsabilité de gestion de certains organismes. Une première percée est tentée lorsque, en décembre 1966, le Commissaire au plan, FrançoisXavier Ortoli, se voit confier une mission par le Premier ministre «suite aux craintes que les organisations syndicales ont exprimées devant les conséquences sociales de l'évolution des structures de l'économie» (OrToli, 1967, p. 1). Son rapport propose notamment la création d'un Office national de l'emploi qui regrouperait les services de placement et d'orientation, de formation professionnelle des adultes et la gestion des actions du FNE. Le rapporteur précise qu'il a "été tenté de proposer la constitution d'un office tripartite, unissant l'État, les organisations patronales et les organisations syndicales» (ibidem, p. 47), mais que des consultations l'ont convaincu que le dossier n'était pas mûr. Il suggère qu'il soit réexaminé en 1970. Cependant, même le projet moins ambitieux d'Office national de l'emploi déclenche des oppositions conjointes qui l'emporteront rapidement. D'une part, les

(9) Les fonctions exercées par le Plan au cours des «Trente Glorieuses» ont fait l'objet de nombreuses études et controverses dans lesquelles il n'est pas possible d'entrer ici. Notre analyse se limite à ses interventions dans le domaine de l'emploi.

(10) Ce constat résulte de nombreux entretiens menés au début des années 1970 avec des membres de ces comités. services du ministère du Travail, et tout spécialement le corps de l'inspection du travail, combattent ce qu'ils jugent être un risque de démembrement ou de dénaturation des fonctions de l'État. D'autre part, certaines organisations syndicales et patronales, en particulier la CGT-FO et le CNPF, sont très attachées à la défense de l'indépendance des institutions paritaires qu'elles ont fondées (UNEDIC et ASSEDIC). Le projet est enterré. L'ANPE est créée, en 1967, comme établissement public directement soumis à l'autorité du ministre du Travail.

Pour la période que nous étudions, la seule exception notable est constituée par l'Association pour la formation professionnelle des adultes (AFPA). Cette association de droit privé est dirigée par une assemblée générale et un bureau de composition tripartite. L'expérience semble enseigner que dans un organisme dont la quasi-totalité des ressources est fournie par l'État, l'intervention des acteurs sociaux ne relève guère que du registre «consultatif fort». L'État serait embarrassé de se heurter à une coalition majoritaire de ces derniers, en particulier sur le vote du budget. Il accepte, en général, les concessions nécessaires pour l'éviter, ce qui, compte tenu du pluralisme des acteurs sociaux, ne constitue pas pour lui une contrainte très redoutable (11).

S'il existe une tendance au partage des responsabilités en matière de politique de l'emploi entre l'État et les acteurs économiques et sociaux, ce ne sont donc pas les diverses formes de tripartisme qui en fournissent les illustrations principales.

\section{La contractualisation}

Un saut qualitatif(12) dans les modes de mise en œuvre de la politique de l'emploi est opéré avec la loi du 18 décembre 1963 qui crée le FNE (Villebrun, 1965; Thomas, Balmary, 1969; Colin, 1984). Bien qu'il ne s'agisse formellement que d'un regroupement, dans le cadre du ministère du Travail, de lignes budgétaires jusqu'alors dispersées dans différents ministères ou organismes, cette

(11) La même observation sera valable, dans la période ultérieure, pour l'ANPE dotée, à partir de 1980, d'un conseil d'administration tripartite.

(12) Il est toujours quelque peu arbitraire de fixer la date d'une inflexion significative. Dès 1961, une réorganisation de la Direction générale du travail et de la main-d'œuvre (DGTMO), est décidée, avec en son sein une division de la coordination. En 1962, les premiers échelons régionaux de l'emploi et du travail (ERET) sont créés (MAClouf, 2006). Cependant, 1963 apparaît comme l'année décisive avec d'une part, la création du FNE, qui transforme la nature de l'intervention publique, d'autre part, le «plan de stabilisation» mis en œuvre sous l'impulsion de Valery Giscard d'Estaing, alors ministre des Finances. Ce plan recherche une «détente» sur le marché du travail et ouvre des controverses durables sur la nécessité d'un «volant de chômage» pour combattre l'inflation, créant ainsi un contexte macro-économique nouveau pour la politique de l'emploi. 
innovation prend progressivement une place essentielle, qu'elle a conservée aujourd'hui. Un dispositif unifié finance deux types d'interventions du ministère. D'une part, il permet d'attribuer des allocations individuelles pour favoriser la formation et la mobilité géographique de demandeurs d'emploi ou de salariés menacés par un licenciement collectif. D'autre part, il permet à l'État de passer des conventions avec des organisations professionnelles ou des entreprises pour organiser la conversion professionnelle et le reclassement des travailleurs dans des territoires ou des branches d'activité «atteints ou menacés d'un grave déséquilibre de l'emploi».

Dans cette seconde hypothèse, l'État n'agit plus par des opérations ou des prestations décidées unilatéralement, mais par des conventions qu'il passe avec des acteurs économiques ou sociaux et qui constituent la condition nécessaire de son intervention. Ce mécanisme a un double impact:

- en premier lieu, cette modalité d'action de l'État ne peut se réaliser que dans le cadre de "conventions librement négociées », comme le précise le texte final de la loi, après les inquiétudes qu'avait soulevées une première rédaction jugée ambiguë. Il s'agit donc d'une action conjointe de l'État et d'un acteur privé dans le cadre de la politique de l'emploi;

- en second lieu, le mécanisme ainsi introduit ne constitue pas seulement une autolimitation de la liberté d'action de l'État. Grâce aux moyens financiers qu'il met à la disposition du cosignataire, l'État dispose aussi d'une capacité de négociation qui lui permet de peser sur le comportement de ce dernier en matière de gestion de l'emploi. Concrètement, comme ces conventions concernent principalement des opérations de réduction d'effectifs, l'Etat pose des conditions sur l'ampleur des licenciements et sur les garanties et prestations qui sont offertes par l'entreprise aux travailleurs licenciés.

Au départ, la loi privilégiait des conventions avec des organismes à caractère collectif et ne mentionnait que comme éventualité secondaire la possibilité de conventions avec les entreprises (13). En pratique, le cas général devient l'exception et la quasi-totalité des conventions sont passées avec les entreprises parce que c'est à ce niveau que sont décidés et gérés les licenciements collectifs (ThOMAS, BALMARY, 1969). Cependant, à partir de 1966, la tendance, pour les secteurs les plus touchés, sera d'inscrire les conventions d'entreprises dans le cadre d'un accord passé par l'État avec l'organisation patronale de la branche et/ou d'un accord collectif de branche qui fixe un cadre-type pour les futures conventions FNE.

(13) La loi prévoit que des conventions peuvent être passées avec « des organisations professionnelles, interprofessionnelles d'employeurs ou de travailleurs et, le cas échéant, avec des entreprises".
L'illustration principale est donnée par la sidérurgie (REynAud, 1969; Sellier, 1970). Des restructurations massives s'imposant dans la sidérurgie lorraine, la chambre syndicale de la sidérurgie élabore un plan professionnel. Sur cette base, elle négocie avec le Gouvernement une convention Étatprofession (juillet 1966) qui contient des «dispositions relatives aux travailleurs». La mise en œuvre de ces dispositions est renvoyée à une négociation directe entre employeurs et syndicats. Le fractionnement en deux étapes a pour objet d'exclure les syndicats des aspects économiques de la négociation et de limiter leur champ d'intervention à la seule gestion des conséquences sociales. Au terme d'un grave et long conflit, une convention sociale de la sidérurgie lorraine est signée en juin 1967(14). Elle définit avec précision les modalités de réduction des effectifs et les modes de recours au FNE. L'expérience servira de référence pour les politiques ultérieures de gestion des crises sectorielles.

Cet exemple met en évidence la nécessité d'une articulation entre, d'une part, les négociations de conventions de type FNE qui concernent l'État et les employeurs et, d'autre part, la négociation collective sur l'emploi entre organisations patronales et syndicales.

\section{La négociation collective et les institutions paritaires}

Le développement de la négociation collective, à partir de la loi de 1950 qui en élargit le champ de compétence, fait des organisations patronales et syndicales de nouveaux acteurs potentiels de la politique de l'emploi, en complémentarité ou en concurrence avec l'intervention publique. Ils seront longs à se saisir pleinement de cette possibilité. Deux dates sont déterminantes en de domaine: 1958, avec la création de l'assurance chômage, et 1968, avec le projet de protocole d'accord de Grenelle qui ouvre plusieurs champs nouveaux d'intervention.

\section{L'assurance chômage et ses extensions}

Il est exclu ici d'entrer dans l'histoire complexe du régime paritaire d'assurance chômage (DANIEL, 1997; Daniel, Tuchszirer, 1999; Gau, 1963; UNEDIC, 1983). Nous n'aborderons que deux aspects: d'une part, la complexité des liens avec l'Etat, d'autre part, l'indétermination de la frontière,

(14) La revue Droit social a publié le texte de cette convention auquel est jointe une "Convention type de coopération avec le Fonds national de l'emploi» (Droit social, juin 1969, p. 371379). La CGT, initialement non signataire, adhère à l'accord en janvier 1968 pour ne pas être exclue de ses dispositifs de mise en œuvre, notamment de la «Commission de l'emploi» créée par l'accord à cette fin. 
en ce domaine, entre protection sociale et politique de l'emploi.

Si les négociateurs et les gestionnaires du régime d'assurance chômage affirment fréquemment et avec force leur indépendance à l'égard de l'État, il s'agit plus d'une pétition de principe que d'un constat objectif. Certes, la création de l'UNEDIC a été précédée de discussions, dès 1957, entre le CNPF et la CGT-FO, mais la négociation ne démarre réellement qu'après la ferme invitation adressée le premier août 1958 par le général de Gaulle, nouveau président du Conseil, aux «partenaires sociaux». Si l'accord est daté du 31 décembre de la même année, c'est que telle était la date limite fixée avant une intervention directe du Gouvernement. Rappelons enfin que, dès l'origine, les accords interprofessionnels en matière d'assurance chômage n'ont été valables qu'après leur agrément par l'État (15).

En 1963, lorsque le projet de loi portant création du FNE est examiné, c'est de l'UNEDIC que viennent les réactions les plus négatives. Ses gestionnaires craignent que par ce biais l'État n'impose des ponctions obligatoires sur ses excédents. En 1967, comme nous l'avons vu, c'est la résistance de la majorité des organisations patronales et syndicales qui conduit le rapport Ortoli à repousser le projet de création d'un "grand service de l'emploi à gestion tripartite». Cependant, en juillet de la même année, c'est par ordonnance que sont réduites les incohérences entre régime paritaire d'assurance et régime public d'assistance.

Dès l'origine, les caractéristiques du régime d'assurance chômage sont déterminées par deux procédures enchevêtrées: d'une part, une négociation collective interprofessionnelle entre organisations patronales et syndicales, d'autre part, des discussions, ouvertes ou cachées, entre l'État et les négociateurs (ou une partie de ces derniers). L'agrément final porte sur un texte qui traduit les interactions entre les deux procédures.

Cette première source d'indétermination (paritarisme ou tripartisme asymétrique masqué ?) est étroitement dépendante d'une seconde, celle qui porte sur la légitimité d'une intervention de l'UNEDIC, au-delà de l'indemnisation des chômeurs, dans des dispositifs de la politique de l'emploi. Le problème se pose très tôt car la conjoncture économique, plus favorable qu'il n'était prévu en 1958, engendre d'importants excédents. Au sein de l'UNEDIC, les avis sont partagés avec, aux extrêmes, d'une part, la CGT-FO, qui défend une politique de pure indemnisation et renvoie à l'État la responsabilité de la politique de l'emploi, d'autre part la CFTC (elle devient en 1964 la CFDT) qui considère légitime et

(15) Le Code du travail fait de l'indemnisation du chômage une responsabilité de l'État que l'UNEDIC n'exerce, pour partie, que par délégation. souhaitable le financement d'actions préventives ou curatives contre le chômage. L'appareil d'État est tout aussi divisé: l'administration du ministère du Travail défend sa pleine compétence dans la politique de l'emploi, tandis que celle des finances voit d'un bon œil une participation financière (et donc accepte un droit d'initiative) de l'UNEDIC.

Si les arbitrages sont difficiles, ils aboutissent à des engagements non négligeables de l'UNEDIC. En créant, dès 1961, une allocation de formation pour les chômeurs qu'elle indemnise l'UNEDIC pénètre dans le domaine des politiques actives de l'emploi. Surtout, l'UNEDIC est amenée, dans le cadre de l'accord interprofessionnel du 27 mars 1972, à financer une garantie de ressource - licenciement au bénéfice de travailleurs licenciés après 60 ans. Cet accord a une importance considérable et durable (GuilLEMARD, 1983). Il traduit un compromis difficile entre, d'une part, les organisations syndicales, principalement la CFDT et la CGT, qui revendiquent le droit à la retraite à taux plein à soixante ans, et, d'autre part, le patronat, qui refuse une concession jugée irréversible, mais cherche, pour des raisons de coût et de productivité, à se débarrasser des salariés âgés. Avec l'appui de l'État, le patronat fait accepter une solution qui attribue un revenu de substitution aux seuls travailleurs âgés licenciés : le régime s'engage ainsi dans la gestion sociale des restructurations industrielles aux côtés de l'État et avec un dispositif de portée générale alors que les interventions du FNE sont décidées au cas par cas.

Ainsi, avant même la période de chômage massif, l'UNEDIC apparaît comme financeur et comme décideur (ou codécideur) dans des domaines importants de la politique de l'emploi.

\section{Le protocole de Grenelle et ses suites}

Le protocole d'accord de Grenelle est resté juridiquement à l'état de projet. Cependant, il a exercé une forte influence sur la négociation collective des années suivantes dans la mesure où il n'était pas seulement le reflet d'un rapport de forces conjoncturel, mais où il contenait une sélection des domaines dans lesquels les organisations patronales et syndicales avaient identifié des champs de compromis possibles. Parmi les questions traitées, trois relèvent directement de la politique de l'emploi: la réduction de la durée du travail, la sécurité de l'emploi, la formation et le perfectionnement professionnels. Nous ne traiterons pas de la durée du travail, domaine dans lequel l'État a, de fait, laissé la main à la négociation collective jusqu'en 1981(16). L'accent sera mis sur les deux autres points où les interactions avec l'État sont plus nettes.

(16) Nous renvoyons sur ce point à une autre contribution, FREYSSINET, 1997. 


\section{L'accord sur la sécurité de l'emploi}

Le projet de protocole prévoit l'ouverture de négociations «en matière de sécurité de l'emploi portant notamment» sur les mesures de reclassement en cas de fusion et de concentration d'entreprises et sur la création de commissions paritaires de l'emploi par branches. Après des négociations difficiles, un accord est signé le 10 février 1969 (Delamotte, 1969; Loos, 1983). Sans examiner le détail de son contenu, plusieurs observations s'imposent:

- toutes les organisations syndicales sont signataires, ce qui est rare. Elles manifestent ainsi l'importance qu'elles attachent à une prise de responsabilité dans le domaine de la gestion de l'emploi;

- c'est la première fois que le CNPF signe un accord interprofessionnel en dehors du champ de la protection sociale, ce qu'il avait toujours refusé jusqu'en mai 1968, en renvoyant à la négociation de branche. Toutefois le CNPF a exclu, sauf sur des points mineurs, d'élargir la négociation au-delà des deux thèmes mentionnés, après le terme "notamment", dans le protocole de Grenelle (17);

- dans les deux cas, l'accord s'appuie sur des accords de branche antérieurs, en particulier celui de la sidérurgie. Il s'agit d'un accord de méthode plus que d'un accord de contenu; il fixe des orientations générales dont les accords de branche futurs devront préciser la mise en œuvre;

- un des facteurs d'obtention du compromis a résulté des engagements qu'a pris le Gouvernement, à la demande des négociateurs et alors que la négociation était en difficulté, sur l'intervention du FNE et sur la formation professionnelle. Ainsi s'établit une articulation directe entre dispositifs négociés et instruments de la politique publique de l'emploi.

\section{L'accord sur la formation et le perfectionnement professionnels}

Le protocole de Grenelle ne prévoyait que «d'étudier les moyens permettant d'assurer, avec le concours de l'État, la formation et le perfectionnement professionnels». Jusqu'alors l'État avait seul compétence générale en ce domaine. Si des négociations interprofessionnelles s'ouvrent sur ce sujet et aboutissent à l'accord du 9 juillet 1970 (18), l'imbrication avec l'intervention publique est forte, d'abord de manière informelle mais permanente

(17) En particulier, la CFDT et la CGT ont échoué dans leur objectif d'introduire le principe: «Pas de licenciement sans reclassement assuré».

(18) L'accord est signé, côté syndical, par quatre confédérations, à l'exclusion de la CGC. Cette dernière signera, avec elles, un avenant ultérieur relatif au personnel d'encadrement. La bibliographie relative à la loi et à l'accord est considérable; nous nous bornons à renvoyer à quelques textes directement liées à notre problématique, Delors, 1991 et 2004, (chapitre 3); Droit social, 1973; MériauX, 1997; VInCENT, 1997. Pour une mise en perspective historique: LUTTRINGER, 1986. au cours de la négociation, ensuite avec la loi du 16 juillet 1971 qui élargit les résultats de l'accord. Celui-ci porte principalement sur le droit au congé individuel de formation et sur le recours à la formation pour les salariés menacés ou victimes de licenciement économique. La loi innove principalement en créant, pour les employeurs, une obligation minimale de dépense au titre de la formation continue. Le lien étroit entre accord professionnel et loi restera une caractéristique durable en matière de formation professionnelle continue, au point que le concept de «loi négociée» sera imaginé sur cet exemple (VERDIER, LANGLOIS, 1972).

Par des canaux divers, l'État a été conduit, au travers de multiples réformes partielles, à reconnaître aux acteurs économiques et sociaux un champ de responsabilité d'ampleur croissante, non seulement dans l'orientation de la politique de l'emploi, mais aussi dans sa mise en œuvre. L'évolution n'a pas engendré un partage clair des compétences, mais un empilement d'institutions et de dispositifs hétérogènes. Aussi longtemps qu'une croissance forte et un chômage faible facilitaient la définition et le financement des compromis, cet échafaudage complexe parvenait à fonctionner, au prix de quelques incohérences et de gaspillages de ressources, mais sans conflits majeurs ni dysfonctionnements socialement dangereux. Le système révélera les limites de son efficacité lorsque s'effondrera le modèle de croissance qui avait servi de base à son édification.

\section{Après 1973, ruptures et continuités}

Il est souvent considéré que, si la décennie 1970 a été marquée par une rupture brutale dans la fonction et le contenu des politiques de l'emploi, les institutions et acteurs responsables de leur mise en œuvre sont restés remarquablement stables.

La première affirmation parait s'imposer comme une évidence. L'apparition d'un chômage massif et persistant entraîne un considérable accroissement de la dépense publique pour l'emploi(19). Des dispositifs nouveaux sont successivement mis en place en direction des catégories les plus durement touchées (jeunes, travailleurs âgés, chômeurs de longue durée, titulaires de minima sociaux...). D'autres sphères de l'action publique sont mises au service de la politique de l'emploi («assouplissement» de la législation du travail, exonérations de charges sociales patronales, professionnalisation des forma-

(19) Selon les définitions adoptées par la DARES, la dépense publique pour l'emploi passe de $0,9 \%$ du produit intérieur brut en 1973 à $4,7 \%$ en 2003, tandis que le nombre de personnes touchées par les différents dispositifs passe de 132000 en 1974 à 2,2 millions en 2004. 
tions initiales...). La politique de l'emploi a donc connu un développement de son champ d'action, de ses ressources et de ses outils en même temps qu'elle était rangée, au moins dans le discours, aux premiers rangs des priorités de l'État (20).

Ce constat rend surprenante l'apparente stabilité des configurations d'acteurs et des institutions intervenant dans la politique de l'emploi. Tous ou presque étaient en place dès le début des années 1970, pour autant que l'on n'observe que le niveau national. Les seuls changements notables résultent de la création au ministère du Travail, en 1975, de la Délégation à l'emploi, issue du fractionnement de la Direction générale du travail et de la maind'œuvre, puis, en 1985, de la Délégation à la formation professionnelle, issue de services jusqu'alors interministériels. Ces deux délégations sont fusionnées en 1997 pour constituer l'actuelle Délégation générale à l'emploi et à la formation professionnelle. Les instances de consultation nationales et territoriales, ainsi que le FNE, l'ANPE, l'AFPA ou l'UNEDIC (devenue Unédic) n'ont pas connu de changements institutionnels notables.

En revanche, des mutations, potentiellement profondes, ont été engendrées par le développement des compétences d'échelons supra et surtout infranationaux.

Les diagnostics sont partagés quant à la force et à la nature de l'impact exercé sur les politiques nationales par la Stratégie européenne de l'emploi. S'il est incertain et ambigu, il n'est certainement pas négligeable (21).

La source de transformation la plus manifeste résulte de la politique de décentralisation et de l'attribution aux collectivités locales, par des canaux indirects, de responsabilités en matière d'emploi : les régions, à travers leurs compétences en matière de développement économique et de formation professionnelle, les départements (et plus récemment les communes) à travers leurs compétences en matière de politique sociale.

Comme, dans le même temps, les institutions paritaires, qu'il s'agisse de l'assurance chômage ou de la formation professionnelle continue, ont élargi leur prise en compte des questions d'emploi et renforcé leur volonté d'y intervenir, la configuration d'acteurs a acquis un niveau exceptionnel de complexité, bien décrit par le rapport de Jean Marimbert (MARIMBERT, 2004).

(20) Pour des synthèses sur la politique de l'emploi durant cette période, avec des bibliographies, voir par exemple, Colin et alii, 1981; DARES, 2003; Elbaum, 1997; FREYsSINET, 2004.

(21) Dans la période antérieure, l'influence européenne ne s'exerçait que très indirectement par l'intermédiaire du Fonds social européen (FSE), créé en 1957. Il s'agissait surtout pour les responsables nationaux de présenter leurs projets de matière telle qu'ils satisfassent les critères d'admission à un cofinancement du FSE.
Les instances de consultation demeurent, avec des adaptations mineures et une influence toujours aussi incertaine, dans le cadre du ministère du Travail, tandis que la concertation a souffert de l'effacement progressif, puis de la suppression du Commissariat général du plan.

La contractualisation assurée par l'intermédiaire du FNE porte sur des volumes financiers croissants et s'opère toujours principalement avec les entreprises dans le cadre des restructurations. Une nouvelle modalité s'est développée avec le recours croissant à la sous-traitance avec des opérateurs privés (BALMARY et alii, 2004) pour la mise en œuvre des prestations de la politique de l'emploi (22).

La procédure de conventionnement a été imaginée pour coordonner, avec de grandes difficultés, les objectifs et les interventions des principaux acteurs au niveau national et territorial(23).

Enfin, les collectivités territoriales, jalouses de compétences récemment acquises, voient avec réticence toute tentative de coordination qu'elles interprètent comme des tentatives de reprise du contrôle par l'État.

Si la gouvernance de la politique de l'emploi est toujours, dans les textes, sous la responsabilité de l'État, elle se présente, en fait, comme une configuration multipolaire d'acteurs faiblement coordonnés. Cette tendance avait émergé dans la période antérieure. Elle a pris aujourd'hui une force prédominante.
(22) Comme les collectivités territoriales et l'Unédic pratiquent la même politique, le schéma ne se simplifie pas.

(23) La convention État-ANPE-Unédic fournit une bonne illustration de la difficulté et des ambiguïtés de ces modes de coordination. 


\section{Bibliographie}

Balmary D., Chevrier-Fatome C., Simonin B. (2004), Politique de l'emploi et recours à des opérateurs externes, Commissariat général du plan, Paris, La Documentation française.

Benoît A. (1984), «La politique de l'emploi. Organisation et moyens», Notes et études documentaires, n ${ }^{\circ} 4752$, Paris, La Documentation française.

BÉRANGER T. (1975), «1959-1974 : quinze ans d'assurance chômage», Droit social, septembre-octobre, p. 135-141.

CGP (1947), «Rapport de la Commission de la maind'œuvre - I ${ }^{\text {er }}$ Plan », Revue française du travail, janvier, p. $32-53$.

CGP (1954), «Rapport général de la Commission de la main-d'œuvre - II ${ }^{\mathrm{e}}$ Plan», Revue française du travail, $\mathrm{n}^{\mathrm{o}} 3$, p. 1-158.

CGP (1958), «Rapport général de la Commission de la main-d'œuvre - III ${ }^{\mathrm{e}}$ Plan», Revue française du travail, avril-juin, p. 3-264.

CGP (1961), «Rapport général de la Commission de la main-d'œuvre pour le IV e Plan de développement économique et social», Revue française du travail, octobre-décembre, p. 1-191.

CGP (1966), Rapport général de la Commission de la main-d'œuvre - Ve Plan 1966-1970, Paris, La Documentation française.

CGP (1971), Rapport de la Commission emploi. Préparation du VI ${ }^{\mathrm{e}}$ Plan 1971-1975, deux volumes, Paris, La Documentation française.

Chatriot A., Join-Lambert O., Viet V. (2006), Les politiques du travail (1906-2006). Acteurs, institutions, réseaux, Presses universitaires de Rennes.

Chazelle J. (1964), Rapport pour une rénovation des services de l'emploi, ministère du Travail, Direction générale du travail et de l'emploi, juin (non publié).

Colin J.-F. (1984), «Le Fonds national de l'emploi 19631983 », in: Comptes de l'emploi. Données physico-financières 1980-1982, Paris, La Documentation française.

Colin J.-F., Cros J.-C., Verdier E., Welcomme D. (1981), «Politiques d'emploi: la rupture de $1977 »$, Travail et Emploi, octobre-décembre, p. 9-22.

Dahano R. (1971), «Politique de l'emploi et lutte contre l'inflation», Revue française des affaires sociales, octobre-décembre, p. 13-28.

DANiEl C. (1997), «La naissance du régime conventionnel d'assurance-chômage en 1958 ou les ambiguïtés du paritarisme à la française », La revue de l'IRES, $\mathrm{n}^{\circ} 24$, p. 131-152.

DAniel C., Tuchszirer C. (1999), L'État face aux chômeurs. L'indemnisation du chômage de 1884 à nos jours, Paris, Flammarion.
DARES (2003), Les politiques de l'emploi et du marché du travail, Paris, La Découverte.

Dassetto B. (1972), «La Commission de l'emploi du VIe plan», Droit social, avril-mai 1972, p. 94-101.

Decouflé A.-C. (1982), «Éléments d'introduction à une histoire des politiques du travail et de l'emploi», Travail et Emploi, $\mathrm{n}^{\circ} 11$, p. 83-89.

Decouflé A.-C. (1985), Les politiques du travail et de l'emploi en France, 1791-1981. Étude bibliographique, Paris, La Documentation française.

Decouflé A.-C. (1988), «Les modes de formation historique des politiques de l'emploi en France», in: ministère d'État chargé du Plan et de l'Aménagement $\mathrm{du}$ territoire, ministère du Travail, de l'Emploi et de la Formation professionnelle, Structures du marché du travail et politiques de l'emploi, Paris, SyrosAlternatives.

Decouflé A.-C. (1990), Quarante ans de politique du travail et de l'emploi en France (1946-1986). Éléments pour un bilan critique, Paris, Ed. Matrice.

Delamotte Y. (1969), «L'accord interprofessionnel sur la sécurité de l'emploi du 10 février $1969 »$, Droit social, septembre-octobre, p. 498-507.

Delors J. (1991), «Genèse d'une loi et stratégie de changement», Formation et Emploi, no 34 , p. 1-38.

Delors J. (2004), Mémoires, Paris, Plon.

Desmarets J. (1946), La politique de la main-d'œuvre en France, Paris, PUF.

Droit social, 1973, «La formation professionnelle continue», numéro spécial, septembre-octobre.

Elbaum M. (1987), «Les politiques de l'emploi depuis trente ans », in INSEE, Données sociales, Paris.

FREYSSINET J. (1997), Le temps de travail en miettes. Vingt ans de politique de l'emploi et de négociation collective, Paris, éditions de l'Atelier.

Freyssinet J. (2004), «Agir pour l'emploi. Quelques enseignements de l'expérience», Projet, n 278.

Freyssinet J. (2006), «L'émergence des politiques de l'emploi (1945-1973)», in Chatriot et alii, 2006, et sous le même titre: Document de travail, $\mathrm{n}^{\circ} 65$, Centre d'études de l'emploi.

GAU J.-A. (1963), «Résultats et tendances du régime conventionnel», Droit social, mars, p. 170-186.

GiLlard L., (1974), «La politique sélective de l'emploi : pratiques suédoises et problèmes théoriques», Revue française des affaires sociales, octobre-décembre, p. 131-152.

Guillemard A.-M. (1983), «La dynamique sociale des cessations anticipées d'activité», Travail et Emploi, $n^{\circ} 15$, p. 15-31. 
HAYWARDJ. (1967), «Lefonctionnement des Commissions et la préparation du Ve Plan: l'exemple de la Commission de la main-d'œuvre», Revue française de sociologie, octobre-décembre, p. 447-467.

JACHIET N. (1981), «La planification française et l'emploi », Travail et Emploi, no 7, janvier, p. 7-20.

Join-Lambert M.-T., Bolot-Gittler A., Daniel C., Lenoir D., MéDA D. (1997), Politiques sociales, deuxième édition, Paris, Presses de Sciences-Po et Dalloz.

Lacroix B., Merle V. (1988), «Les politiques de l'emploi et le fonctionnement des institutions publiques », in: ministère d'État chargé du Plan et de l'Aménagement du territoire, ministère du Travail, de l'Emploi et de la Formation professionnelle, Structures $d u$ marché du travail et politiques de l'emploi, Paris, SyrosAlternatives.

LAURENT P. (1963), «Les aspects nouveaux de la politique de l'emploi », Revue française du travail, avril-juin, p. 9-21.

Loos J. (1983), «Accord de contenu ou accord de méthode? L'exemple de l'accord national interprofessionnel de 1969 sur la sécurité de l'emploi», Sociologie du travail, premier trimestre, p. 15-31.

LutTringer J.-M. (1986), Le droit de la formation continue, Paris, Dalloz.

Maclouf P. (2006), "“L'emploi” du travail (19142006)», in CHATRIOT et alii, 2006.

MARimbert J. (2004), «Rapport au ministre des Affaires sociales, du travail et de la solidarité sur le rapprochement des services de l'emploi», janvier 2004.

Massé P. (1964), «Les problèmes de l'emploi dans les perspectives du Ve Plan», Revue française du travail, avril-juin, p. 19-34.

Merle V. (1987), «Transformations du marché du travail et transformation de l'intervention publique», Les Temps Modernes, novembre-décembre, p. 233-259.
MéRIAuX O. (1997), «Vers un nouveau modèle de paritarisme dans la gestion des fonds de la formation professionnelle? », La revue de l'IRES, no 24, p. 191-208.

Ortoli F.-X. (1967), «Rapport sur les conséquences sociales de l'évolution des structures de l'économie», Commissariat général du plan, avril (non publié).

Reynaud J.-D. (1969), «La convention sociale de la sidérurgie lorraine», Droit social, avril, p. 219-227.

Roux R. (1949), «De la politique de la main-d'œuvre à une politique de l'emploi?», Revue française du travail, juillet-août, p. 385-407.

SEllier F. (1970), «L'évolution des négociations collectives dans la sidérurgie et la métallurgie en France (19501969)», Droit social, septembre-octobre, p. 431-442.

SEllier F. (1984), La confrontation sociale en France 1936-1981, Paris, PUF.

Thomas C., Balmary D. (1969), «Le Fonds national de l'emploi», Droit social, décembre, p. 575-585.

UNEDIC (1983), Histoire du régime d'assurancechômage 1959-1982, Paris, UNEDIC.

Université Paris-II (1981), L'administration et l'emploi, Paris, La Documentation française.

Verdier J.-M., Langlois P. (1972), «Aux confins de la théorie des sources du droit: une relation nouvelle entre la loi et l'accord collectif», Recueil Dalloz-Sirey, $39^{\circ}$ cahier, p. 253-260.

VIET V. (2006), «La politique de la main-d'œuvre: un domaine d'action atypique du ministère du Travail et de la Prévoyance sociale», in CHAтRIот et alii, 2006.

Villebrun J. (1965), «La loi du 18 décembre 1963 et le Fonds national de l'emploi », Droit social, février, p. 8796.

VINCENT C. (1997), «De l'accord de juillet 1970 à la loi de 1971: l'échec d'un paritarisme négocié de la formation professionnelle continue», La revue de l'IRES, $\mathrm{n}^{\circ} 24$, p. 153-174. 